\title{
Evidence for a relativistic iron line in GRS 1915+105
}

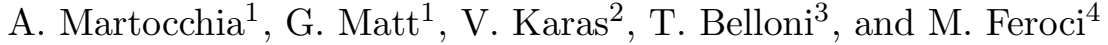 \\ 1 Dipartimento di Fisica, Università degli Studi "Roma Tre", Via della Vasca Navale 84, 00146 Roma, Italy \\ 2 Astronomical Institute, Charles University Prague, Faculty of Mathematics and Physics, V Holešovičkách 2, \\ CZ-180 00 Praha, The Czech Republic \\ 3 Osservatorio Astronomico di Brera, via E. Bianchi 46, 23807 Merate, Italy \\ 4 IAS/CNR, Area di Ricerca di Tor Vergata, Via Fosso del Cavaliere 100, 00133 Roma, Italy
}

Received 6 November 2001 / Accepted 5 March 2002

\begin{abstract}
We report on the discovery of a relativistic iron $\mathrm{K} \alpha$ fluorescent emission line in the BeppoSAX spectrum of the microquasar GRS 1915+105, taken on April 19, 1998, when the line was unusually intense. The feature is broad and skewed, clearly indicating emission from the innermost regions of the accretion disc. The inner emitting orbit is larger than the innermost stable orbit, even in Schwarzschild metric: thus, a non-zero BH spin is not required by these data.
\end{abstract}

Key words. black hole physics - relativity - line: formation - line: profiles - X-rays: individuals: GRS 1915+105

\section{Introduction}

\subsection{Iron line diagnostics of relativistic accretion discs}

Black Hole (BH) accreting systems are expected to exhibit spectral features whose form is determined by reprocessing of primary high-energy photons by gas swirling around the central body. Resulting spectral distortions are of utmost importance because they probe the effects of strong gravity, which are otherwise difficult to reveal. Generalrelativity (GR) calculations of predicted spectra, and their comparison with observational data, may help to measure physical parameters of BHs, such as their masses and angular momenta (e.g. Martocchia 2000, and references therein).

The iron $\mathrm{K} \alpha$ fluorescent emission is specially important as a diagnostic of GR effects (e.g. Fabian et al. 1989; Laor 1991; Matt et al. 1992; Martocchia \& Matt 1996; Martocchia et al. 2000). In case it is originated in the innermost regions of an accretion disc, it gives the possibility, in principle, of distinguishing between a rotating (Kerr) and a static (Schwarzschild) central BH. This is because the resulting line profile is affected both by Special Relativity (Doppler, including transverse, shifts) and General Relativity (energy shift, lensing) effects. It is very broad and asymmetric, the exact shape critically depending on the geometrical parameters of the emitting region (disc inner and outer radii, inclination angle). For the same set of parameters, differences between Schwarzschild

Send offprint requests to: A. Martocchia, e-mail: martocchia@fis.uniroma3.it and extreme Kerr line profiles are rather subtle. However, the innermost stable orbits of the disc are different in the two cases - corresponding respectively to 6 and 1.23 gravitational radii - and the profiles are very different for the two values. The measurement of an innermost emitting radius less than $6 r_{\mathrm{g}}$ can therefore be the signature of a spinning $\mathrm{BH}$.

This diagnostic has been successfully used in the case of Seyfert galaxies (e.g. Tanaka et al. 1995; Wilms et al. 2001), while in galactic BH candidates ionization of the inner accretion flow generally prevents fluorescent emission from being effective. Nevertheless, intense, broad Fe $\mathrm{K} \alpha$ emission has been observed in Cyg X-1 with both BeppoSAX (Frontera et al. 2001) and Chandra (Miller et al. 2002a). Large, possibly relativistic $\mathrm{K} \alpha$ profiles have been detected also in XTE J1748-288 (Miller et al. 2001) and XTE J1650-500 (Miller et al. 2002b).

\subsection{GRS $1915+105$}

GRS $1915+105$ is one of the so-called microquasars, Galactic jet sources with properties similar to those of quasars, but on a smaller, stellar scale. It was extensively studied since its discovery by the WATCH experiment on GRANAT in 1992 (Castro-Tirado et al. 1992). Due to very large interstellar absorption, the optical counterpart of this source was not easily found: only recently, Greiner et al. (2001a) reported results of a spectroscopic analysis in the $H$ and $K$ bands, which suggest that the massdonating star is a K-M III star, i.e. that GRS $1915+105$ belongs to the class of low-mass X-ray binaries. 
Greiner et al. (2001b) used these infrared spectroscopic observations to infer the companion's orbital period which is $\sim 33.5$ days - and determine the mass function $f\left(M_{\mathrm{c}}\right) \simeq 9.5 M_{\odot}$. Since the donor's mass, for K-M III stars of that kind, is known to be $\sim 1.2 M_{\odot}$, and the inclination is known, too, the mass of the central compact object has been constrained to $M_{\mathrm{c}}=14 \pm 4 M_{\odot}$, i.e. well above the standard neutron star mass limit.

Weak variable radio/IR emission connected with jets has been often detected (Mirabel \& Rodriguez 1994). Measurements of the jets' superluminal motion allowed to estimate the source distance $D \sim 12.5 \mathrm{kpc}$ and inclination $i \sim 70^{\circ}$ (Rodriguez \& Mirabel 1999; Fender et al. 1999).

GRS $1915+105$ is well-known for the extremely wide variety of its variability modes, ranging over all possible bands and timescales (e.g. Markwardt et al. 1999; Belloni et al. 1997a,b; Greiner et al. 1996). Time-resolved observations have been intensively performed especially with the Rossi X-ray Timing Explorer (RXTE) satellite mission. Belloni et al. (2000), for instance, managed to classify these observations into 12 separate classes based on count rate and color characteristics. In this way, the source variability could be explained in terms of transitions between three basic states: two soft states with a fully observable disc at two different temperatures (" $\mathrm{A}$ " with the lower temperature, "B" with the higher), and a hard state corresponding to the non-observability of the innermost parts of the accretion disc (state "C"). State "C", with low flux and hard color, can last uninterruptedly for weeks or even months. The most popular model for the source's large scale variability is the one proposed by Belloni et al. (1997a,b), who invoked the onset of a Lightman-Eardley thermal-viscous instability which blows-off the innermost, radiation-pressure supported part of the (optically thick) disc. The hard state $(\mathrm{C})$ would then correspond to a steady replenishment, which takes place on a viscous timescale. Indeed, evidence of the temporary disappearance and subsequent restoring of the inner portion of the accretion disc comes from simultaneous BeppoSAX and Ryle radiotelescope observations (Feroci et al. 1999).

The iron K $\alpha$ emission line in GRS $1915+105$ is usually not very intense, and is therefore difficult to study in detail - not to mention the dramatic spectral variability of the source, which requires selecting short time intervals, so reducing the statistical quality of the spectra. Lee et al. (2001) recently reported that neutral Fe K $\alpha$ emission is likely present in the high-resolution data of a lowhard state, collected with Chandra HETGS, together with Fe photoelectric edges and absorption lines due to highly ionized matter. These features indicate that ionized iron, probably belonging to the accretion disc itself, is present on the line of sight with abundance larger than solar.

Actually, during the several observational campaigns performed by BeppoSAX, the Fe K $\alpha$ line was almost invariably rather weak, with Equivalent Widths $(E W)$ of a few tens of $\mathrm{eV}$ at most. Only in the observation of April 19,1998 , the line was strong enough $(E W \gtrsim 200 \mathrm{eV}$; cp.

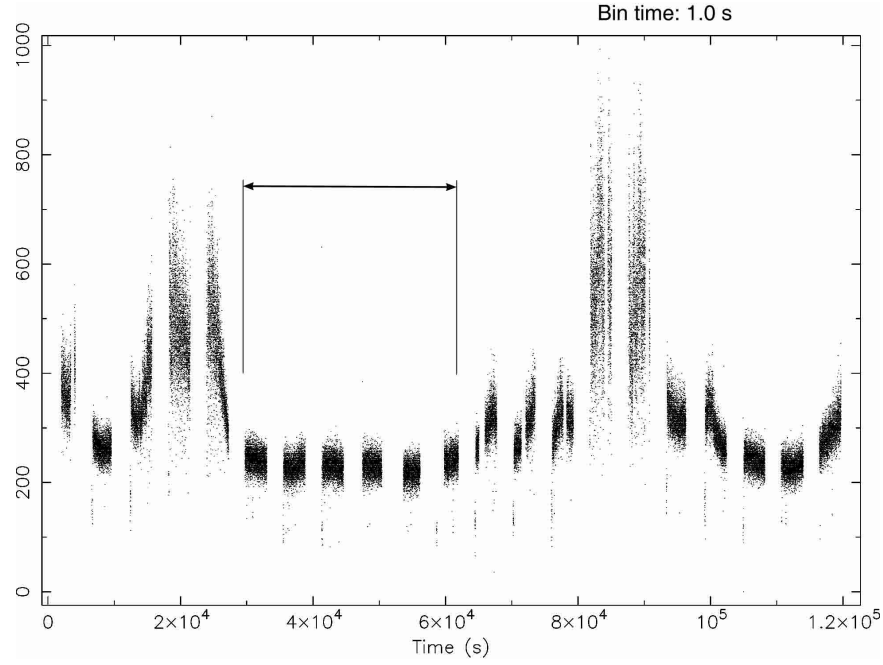

Fig. 1. The MECS lightcurve of GRS 1915+105 during the April 19, 1998 BeppoSAX observation, which lasted 120 ks. The time intervals we considered are those marked (comprised between $t \sim 3 \times 10^{4}$ and $t \sim 6.2 \times 10^{4} \mathrm{~s}$ ). In this paper, the fifth of these intervals is discussed in more detail.

Fig. 3) for a detailed analysis of its profile to be performed. We have therefore analyzed the spectral data from this observation, further selecting some intervals of relative calmness in the lightcurve (see Fig. 1), to limit the spectral variability as much as possible. In order to model the source emission, we adopted the illumination model, local spectra and emissivities described in Martocchia et al. (2001) and Martocchia et al. (2000; see also: Martocchia 2000). Finally, we used our own fitting tool, dedicated to GR spectral distortions in Kerr metric, to analyze the data. The tool, named KerRspec, is described in the Appendix.

\section{Data reduction}

BeppoSAX (Boella et al. 1997) is composed by several instruments. Here we made use of the two imaging instruments, the LECS and the MECS, and the high energy, passively collimated instrument, the PDS. As customary, we have used the following energy bands: $0.1-4 \mathrm{keV}$ for the LECS; $1.8-10 \mathrm{keV}$ for the MECS; $15-150 \mathrm{keV}$ for the PDS (neglecting higher energy data because of insufficient statistics due to the short integration times). The LECS and MECS spectra have been selected from regions of $8^{\prime}$ radius and centered on the source. The background has been evaluated from similar regions in blank field observations, and then subtracted. The PDS is composed by four units, two of which (switching every $96 \mathrm{~s}$ ) monitoring the background, which is automatically subtracted in the pipeline producing the spectrum.

We discuss here the BeppoSAX observation of GRS 1915+105 started on April 19, 1998 at 11:33:19 UT and ended on April 20, 1998 at 20:14:52 UT. Among the several BeppoSAX campaigns on GRS 1915+105, 
we selected this one because the iron line $E W$ was significantly larger than in all other observations.

Out of the entire $\sim 120$ ks observation, some time intervals were sorted out where the source variability is less pronounced. These are the six satellite orbits starting from $3 \times 10^{4} \mathrm{~s}$ after the beginning of the observation, see Fig. 1 . Analysis of the other parts of the observation would require a finer temporal analysis which is beyond the scope of this paper and is deferred to a future work.

All spectral fits were performed with the XSPEC v.11 software package. In the following, all errors refer to $90 \%$ confidence level for one interesting parameter $\left(\Delta \chi^{2}=2.71\right)$.

At the flux level of GRS $1915+105$, it is very likely that systematic errors cannot be neglected with respect to the statistical ones. This may explain the rather large values of the $\chi^{2}$ reported below.

\section{Data analysis and results}

\subsection{The best-fit model in a selected time interval}

Let us first discuss the fifth interval as an example, being the one in which a low value of $\chi^{2}$ was found when fitting with simple models. The model for the continuum includes the usual ingredients for this source: a disc thermal emission (DISKBB), a powerlaw with exponential cutoff, and a Compton Reflection component (PEXRAV: Magdziarz \& Zdziarski 1995), which is expected to always accompany the iron emission line. All these components are seen through interstellar absorption. The best-fit spectrum and $\Delta \chi^{2}$ for the fifth interval, fitting without any iron line, is shown in Fig. 2, left panel $\left(\chi_{\text {red }}^{2}=2.64\right)$. The presence of an iron line is obvious. To better show the profile of the line, we also fitted the spectrum excluding the energy region which contains this feature $(3-7.5 \mathrm{keV})$, and then reintroduced this energy interval. The residuals are shown in Fig. 3 (fifth panel), after reinserting the data of the 3-7.5 keV interval. The presence of a prominent, broad and red-tailed iron line is clear.

We first tried to model the line with a Gaussian. The best-fit is obtained with a rest energy $E_{0}=6.38 \pm$ $0.12 \mathrm{keV}, \sigma=0.70 \pm 0.15 \mathrm{keV}$ and $E W \sim 250 \mathrm{eV}$; the $\chi_{\text {red }}^{2}$ is then 1.48 (see Fig. 2, central panel). The most natural interpretation for a so broad line is emission from a relativistic accretion disc (Fabian et al. 1989), an hypothesis further supported by the asymmetry of the profile (see Fig. 3). The next step was therefore to substitute the Gaussian with the relativistic model KERRSPEC, described in the Appendix. To be self-consistent, we also applied the relativistic corrections to the reflection continuum (using KERRSPEC) as well as to the disc thermal component (using DISKPN, Gierlinski et al. 1999). After having verified, however, that the results are insensitive to the latter corrections, in order to save computer time we adopted the simple, non relativistic models for the continuum in all the following fits. The inclination angle has been always fixed to $70^{\circ}$ (Mirabel \& Rodriguez 1994), while the inner and outer disc radii were kept free to vary. We tried different emissivity laws, and found the best solution with a rather "flat" dependence on radius $\left(\propto r^{-2}\right)$. With the relativistic line model $\left(E_{0}=6.4 \mathrm{keV}\right)$ the fit is better than with a Gaussian: $\chi_{\text {red }}^{2}=1.31$. The spectrum and residuals are shown in Fig. 2, right panel. If $E_{0}$ is instead fixed to $6.7 \mathrm{keV}$, corresponding to He-like iron, the fit is significantly worse $\left(\chi_{\text {red }}^{2} \gg 2\right)$.

We also tried adding a narrow Gaussian centered either at 6.4 or at $6.7 \mathrm{keV}$; no significant improvements in the fits were found.

One problem with the above fits is that the resulting parameters of the thermal emission are rather unplausible. The temperature is very large, $k T=3.77_{-0.43}^{+0.44} \mathrm{keV}$, and the component's normalization very low $(0.41 \pm 0.22$; see Arnaud 1996 for the meaning of this normalization), implying a value of the inner radius of the order of one kilometer. This, in turn, implies an upper limit to the BH mass (obtained assuming the inner emitting radius conciding with the innermost stable orbit for a maximally rotating $\mathrm{BH}$ ) of less than 1 solar mass, contrary to observations (Greiner et al. 2001b). If the disc blackbody component is neglected in the model, the fit e.g. with the Gaussian line yields a worst fit $\chi_{\text {red }}^{2}=1.61$, but the results on the line parameters does not change significantly. Moreover, the fit with a relativistic line is still better than with a Gaussian also in this case.

We therefore conclude that the relativistic solution for the iron line, besides being the most natural physical explanation for the very broad line observed, does not depend on the details of the continuum model.

The small relative luminosity of the disc thermal component (if present at all) brings us to interpret the source's state as a "C"-type, in the scheme of Belloni et al. (2000). This interpretation is in agreement with the occurrence of a "plateau" in the light curve (lower flux and variability), although a hard spectrum would be expected, while instead we get a steep powerlaw, and a cutoff at rather low energies (see Table 1).

\subsection{Other time intervals}

For all other time intervals we found qualitatively similar results: a broad and prominent iron line, which is better fitted by a relativistic line profile than by a Gaussian, and a faint disc blackbody component.

An inspection of the residuals often indicates problems both around $1 \mathrm{keV}$, and above $50 \mathrm{keV}$. The latter problem may be due to a non-thermal component arising when the Comptonized power law cuts-off, while we have no obvious explanation for the former problem. To overcome these difficulties, and because we are interested here to the iron line properties, we decided from now on to exclude the LECS data in the fits, and to use the PDS up to $50 \mathrm{keV}$ only. With this prescriptions, and assuming as working hypothesis that the iron line arises from a relativistic disc, we analysed all the time intervals with a 

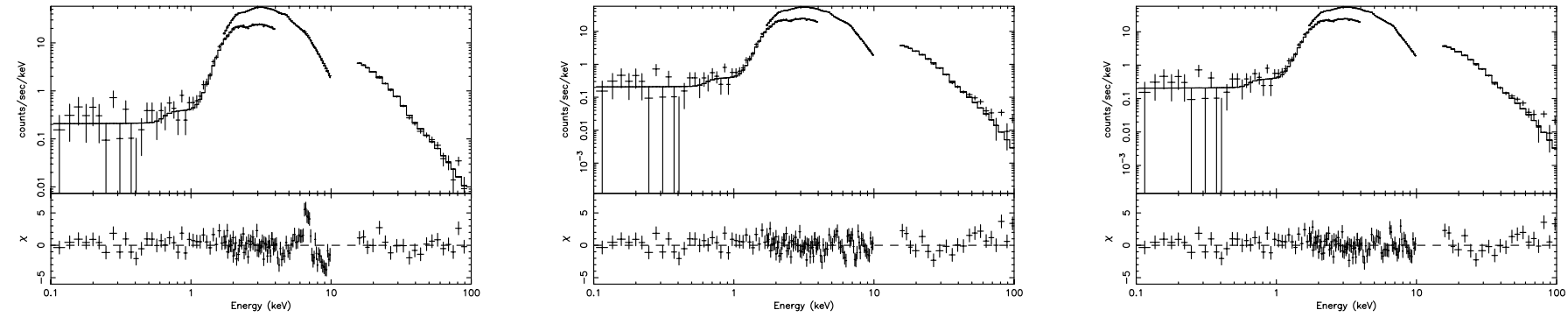

Fig. 2. Left: the best-fit spectrum and $\Delta \chi^{2}$ for the fifth interval, fitting without modelling the iron line $\left(\chi_{\text {red }}^{2}=2.64\right)$ : the residuals are evident. Center: the best-fit spectrum and $\Delta \chi^{2}$ for the same interval, when the line is modelled by a Gaussian profile $\left(\chi_{\text {red }}^{2}=1.48\right)$. Right: the best-fit spectrum and $\Delta \chi^{2}$ for the fifth interval again, with a fully-GR model of the line profile $\left(\chi_{\text {red }}^{2}=1.31\right)$. See the text for details.
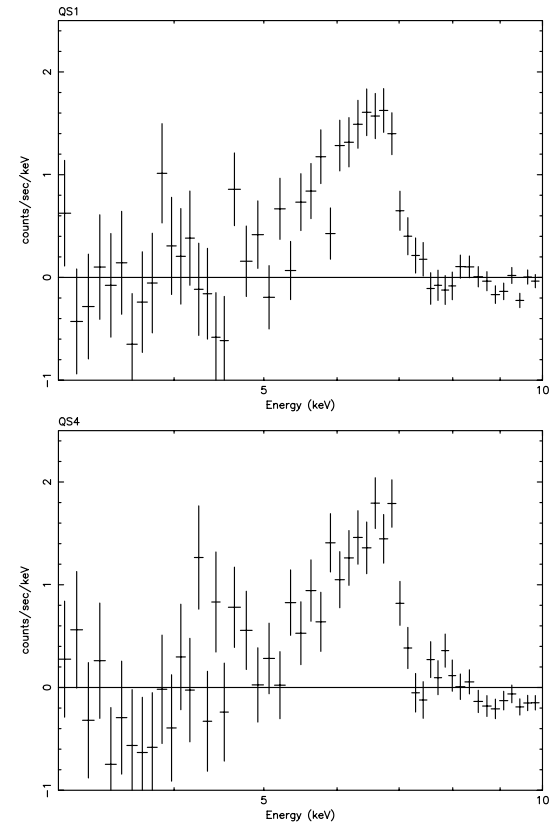
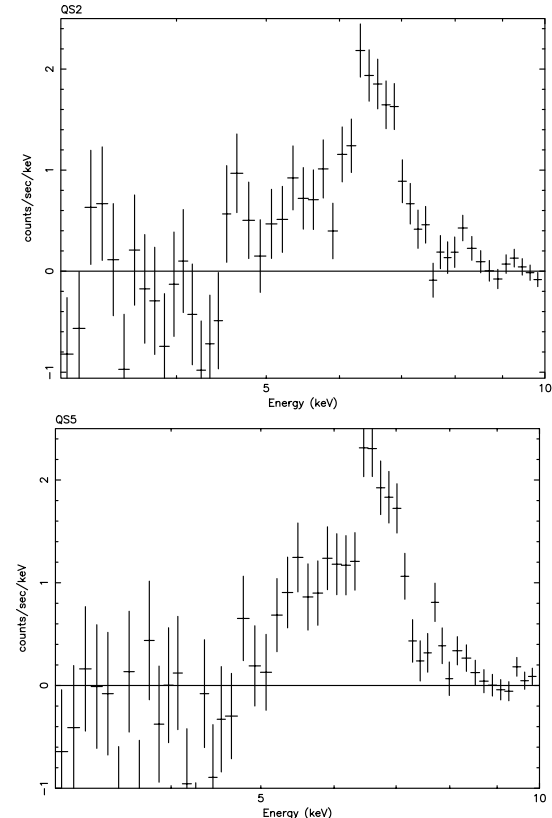
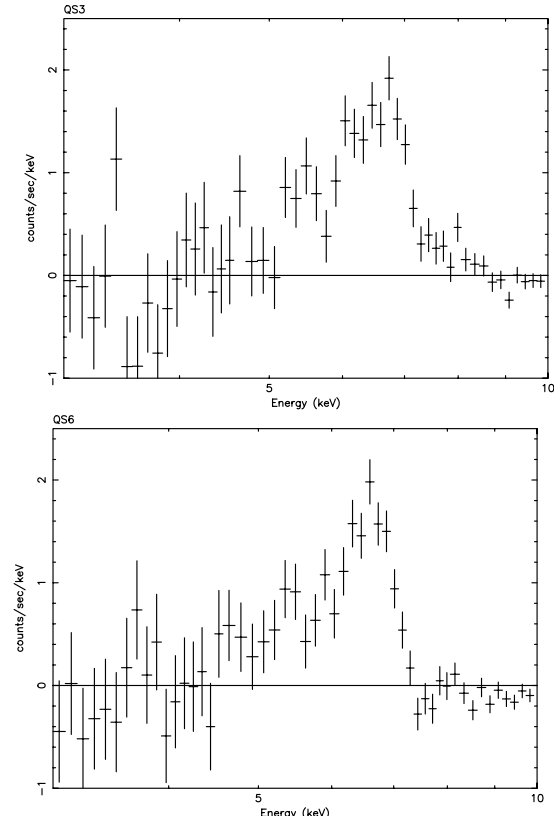

Fig. 3. Residuals at the iron line energies for all the selected time intervals. For illustration purposes, the fits have been performed excluding the energy region of the iron line. Prominent, broad and skewed features are then evident around $\sim 6.4 \mathrm{keV}$.

model which neglects disc thermal emission and includes, besides the line, a power law with exponential cut-off and a Compton reflection continuum. We stress again that the latter component is included more on astrophysical ground (it is supposed to always go along with the iron line) rather than because strongly required by the data. After having verified that the values of the absorbing column densities $n_{\mathrm{H}}$ for the various intervals are all consistent with each another and to the value of $5.4 \times 10^{22} \mathrm{~cm}^{-2}$, for simplicity we fixed in all fits $n_{\mathrm{H}}$ to this value.

The results for the various time intervals are summarized in Table 1 . The larger value of the $\chi_{\text {red }}^{2}$ of the fifth interval with respect to those described above is due to the lower number of spectral bins and to the exclusion of the thermal component. Given the abovementioned uncertainties on the proper modeling of the continuum, we stress that the values in the table should be taken with caution: a different continuum models may result in somewhat different values of the disc parameters. In any case, a general result seems to emerge: the inner radius of the line emitting region is larger than the innermost stable orbit. Whether this means that the innermost regions are absent, or simply that they do not emit the iron line (for instance because the matter is mildly ionized and resonant trapping effective, Matt et al. 1993) we cannot say.

Finally, we tried summing the spectra of all six time intervals together, and fitting with the same model. The very large reduced $\chi^{2}, 5.1$, indicates that spectral variability is too large over these time scales. In particular, strong residuals at the iron line energies imply that the line parameters change with time, as suggested by the results of the time-dependent spectral analysis.

\section{Discussion}

A strong iron $\mathrm{K} \alpha$ fluorescent line is present in the April 19, 1998, spectrum of the superluminal galactic source GRS $1915+105$. The line is broad and skewed, clearly 
Table 1. The best-fit parameters for the various time intervals we considered, excluding LECS data and using a simplified model, which does not take into account thermal emission from the disc (WABS (PEXRAV + KERRSPEC)). The rest line energy is $6.4 \mathrm{keV} . \Delta t$ is the MECS exposure time. See the text for more details.

\begin{tabular}{|c|c|c|c|c|c|c|c|c|c|}
\hline \# interval & $\Delta t$ & $\Gamma$ & $E_{\mathrm{c}}$ & $R$ & $r_{\text {in }}$ & $r_{\text {out }}$ & $E W$ & $F_{2-10}$ & $\chi_{r}^{2}$ \\
\hline & (s) & & $(\mathrm{keV})$ & & $\left(\frac{G M}{c^{2}}\right)$ & $\left(\frac{G M}{c^{2}}\right)$ & $(\mathrm{eV})$ & $\left(10^{-8} \frac{\mathrm{erg}}{\mathrm{cm}^{2} \mathrm{~s}}\right)$ & (68 d.o.f.) \\
\hline 1 & 2562 & $2.638_{-0.012}^{+0.013}$ & $25.6_{-1.2}^{+2.6}$ & $0.21_{-0.20}^{+0.22}$ & $36_{-17}^{+43}$ & $330_{-180}^{+320}$ & $142_{-10}^{+20}$ & 1.876 & 1.27 \\
\hline 2 & 1993 & $2.702_{-0.016}^{+0.017}$ & $26.7_{-2.1}^{+2.4}$ & $0.91_{-0.31}^{+0.34}$ & $79_{-34}^{+51}$ & $710_{-300}^{+610}$ & $192_{-30}^{+25}$ & 1.776 & 1.78 \\
\hline 3 & 2551 & $2.653_{-0.013}^{+0.013}$ & $28.2_{-3.1}^{+2.7}$ & $0.17_{-0.17}^{+0.22}$ & $40_{-24}^{+37}$ & $350_{-110}^{+170}$ & $192_{-23}^{+56}$ & 1.780 & 1.50 \\
\hline 4 & 2003 & $2.666_{-0.014}^{+0.013}$ & $28.8_{-3.4}^{+5.0}$ & $0.60_{-0.09}^{+0.11}$ & $60_{-31}^{+64}$ & $180_{-90}^{+190}$ & $156_{-19}^{+21}$ & 1.799 & 2.58 \\
\hline 5 & 1628 & $2.675_{-0.020}^{+0.023}$ & $23.8_{-1.3}^{+3.9}$ & $1.20_{-0.41}^{+0.49}$ & $7.8_{-3.7}^{+18.4}$ & $240_{-70}^{+100}$ & $298_{-39}^{+30}$ & 1.708 & 1.90 \\
\hline 6 & 2708 & $2.608_{-0.013}^{+0.014}$ & $23.6_{-1.4}^{+5.4}$ & $0.15_{-0.15}^{+0.28}$ & $32_{-13}^{+25}$ & $210_{-110}^{+170}$ & $149_{-22}^{+22}$ & 1.863 & 1.75 \\
\hline
\end{tabular}

indicating emission from a relativistic accretion disc. We successfully fitted the line with a fully relativistic profile. To our knowledge, this is the first time that a relativistic iron line is observed in this source, largely due to the fact that we catched the line with an unusually large equivalent width.

While providing evidence that an accretion disc resides in the innermost part of this system, our result confirms that strong gravity is at work in distorting the iron line profile. From the best-fit parameters we conclude that the line is from neutral or low-ionization iron, and likely emitted from a region of the accretion disc which is close but probably does not include the innermost stable orbit, even for a static BH (but the values of the disc parameters are somewhat dependent on the adopted model for the continuum, and must be taken with caution). This may imply either that the disc does not extend down to this radius, or that the line is not emitted in the innermost regions, maybe because the iron there is in the intervals of ionizations in which resonant trapping efficiently destroys line emission (e.g. Matt et al. 1993, 1996). Unfortunately, at these radii differences in the line profile between Kerr and Schwarzschild metrics are too subtle to be detectable, and the question of the angular momentum of the $\mathrm{BH}-$ which, from this analysis, is not required to have a large value - actually remains open.

So, no final statement about the spin of the $\mathrm{BH}$ in GRS $1915+105$ can still be made. Zhang et al. (1997), on the basis of soft state X-ray brightness (which is most likely due to thermal emission), suggested that a fast rotating $\mathrm{BH}$ is hosted at the center of this source. Their conclusion was based on a mass value twice that measured by Greiner et al. (2001b). However, even account- ing for the correct mass, the BH spin seems to be larger in GRS 1915+105 than in GRO J1655-40, and this does not fit to the results based on QPO diagnostics, as explained below. The accretion disc temperatures of the two sources are nearly identical, and the mass estimate for GRO J1655-40 is $M \sim 7 M_{\odot}$ (Orosz \& Bailyn 1997; Shahbaz et al. 1999). Taking Zhang et al.'s innermost thermally emitting orbit for GRS 1915+105 (at $\sim 40 \mathrm{~km}$, i.e. $r_{\mathrm{ms}} \sim 2 r_{\mathrm{g}}$ ), one infers a BH spin $a \sim 0.95$ in this source (Bardeen et al. 1972). Actually, Zhang et al.'s estimates of the BH angular momentum are based on rather simplified models of the continuum, and they do not include GR spectral distortions and effects of radiation transfer through the disc atmosphere (e.g. Merloni et al. 2000; Gierliński et al. 1999 and 2001). In our data analysis, even accounting for GR effects on the thermal component, we got disc temperatures and luminosities which are physically unplausible, and do not allow us to test assumptions regarding either the $\mathrm{BH}$ mass nor its spin. Non-standard disc models (e.g. slim-disc) could actually reproduce high temperature discs for non-rotating $\mathrm{BHs}$.

QPO diagnostics could therefore be useful as independent tests. Various models try to explain the occurrence of QPOs, suggesting that the oscillations correspond to the motion on the last stable orbit (Shapiro \& Teukolsky 1983), and sometimes invoking GR effects such as "frame dragging" and relativistic precession (e.g. Merloni et al. 1999; Stella et al. 1999). An interesting possibility is that the highest QPO frequency changes in connection with the variation of the disc innermost radius.

QPOs in GRS $1915+105$ have been observed in a wide range of frequencies $(0.001 \div 67 \mathrm{~Hz}$, e.g. Morgan et al. 1997). In GRS 1915+105 the QPO highest frequency 
$(67 \mathrm{~Hz})$, unlike all the others, does not change much with time, and may therefore be related to the accretion disc innermost stable orbit $r_{\mathrm{ms}}$ (Morgan et al. 1997). A similar case is given by the $300 \mathrm{~Hz}$ QPO in GRO J1655-40.

Recently, a second high-frequency QPO has been detected in GRS 1915+105 (Strohmayer 2001), with $\nu_{0} \sim$ $40 \mathrm{~Hz}$. In terms of the frame-dragging model, this lower frequency peak could be associated to the radial epicyclic frequency $\nu_{\mathrm{rad}}$ through the formula $\nu_{0}=\nu_{\mathrm{Kep}}-\nu_{\mathrm{rad}}$ (the nodal precession can be connected to a further lowfrequency peak, already detected at $\sim 0.9 \mathrm{~Hz}$ by Morgan et al. 1997). Strohmayer also reports that the $67 \mathrm{~Hz}$ QPO did in fact drift in frequency by as much as 5 percent. If this frequency is associated with the Keplerian motion at the last stable orbit, then - assuming the BH spin is the same in GRS 1915+105 and GRO J1655-40 - it should scale with $M_{\mathrm{c}}^{-1}$. Thus, a factor $\sim 2 \div 3$ of discrepancy would remain with respect to Greiner et al.'s estimate. This indicates that the BH spin in the two sources is different. If the QPOs in GRS 1915+105 and GRO J1655-40 are seen in the framework of the frame dragging scheme (e.g. Cui et al. 1998, for details on the model), and using Greiner et al.'s mass estimate, we have $a \sim 0.8$ and 0.95 for the two sources, respectively. On the other hand, if the QPO frequency had a diskoseismic origin (e.g. Nowak et al. 1997), the model would require a maximally spinning BH in GRO J1655-40, and an almost static BH in GRS 1915+105 (cp. Greiner et al. 2001b, and references therein).

Clearly, further tests for the BH spin would be of extreme importance. Iron line diagnostics may be crucial to solve the puzzle: better resolution and sensitivity spectral data, provided by satellites of the new (e.g. XMM-Newton) and future (e.g. Constellation-X, XEUS) generations, will be probably decisive in this respect.

Acknowledgements. AM and GM acknowledge financial support from MURST under grant COFIN-00-02-36, GM also from ASI, VK from GAUK 188/2001 and GACR 202/02/0735. TB thanks the Cariplo Foundation for financial support.

\section{Appendix: The computational technique}

We have computed GR effects acting on both the iron $\mathrm{K} \alpha$ line emission and the Compton reflection continuum. We adopt the usual assumption of a geometrically thin, optically thick, cold (with respect to X-rays reprocessing) reflector extending over the equatorial plane. We take into account the directional anisotropy of the illuminating flux, caused by aberration, Doppler effect and light focusing. GR anisotropy of the illuminating flux is crucial to distinguish static from spinning BHs, because the primary illumination, as well as the induced emissivity of the reflection component, carry information about the spacetime in the very vicinity of the $\mathrm{BH}$. The iron line profile and, in particular, its intensity and $E W$ are affected by spacetime distortions (Martocchia \& Matt 1996; Martocchia et al. 2000).
The observed line profiles are often obtained from the data by subtracting the Compton-reflected component without taking into account important relativistic effects, such as the iron edge smearing, which may be relevant if the emission occurs down to the innermost stable orbit, in particular for spinning BH. In our computations (e.g. Martocchia et al. 2000), besides using emissivity laws derived from a proper GR treatment of the illuminating flux, we treated at the same time the GR distortions on the iron line together with the underlying continuum hump (Martocchia et al. 2000; Martocchia et al. 2001).

In order to fit real spectra, a ray-tracing code was developed in the form of a user-defined subroutine, KERRSPEC (Martocchia 2000), which has been linked to the XSPEC data analysis package (Arnaud 1996). In the adopted scheme, a large set of light rays (null geodesics) in the Kerr BH spacetime is pre-calculated and stored. This allows an efficient access during the fitting loop. The code allows the radial, angular and energy dependencies of the local emissivity to be specified on input in a tabular or semi-analytical form. After fixing the desired grid resolution in the image plane, the required light rays corresponding to all image pixels are retrieved.

The original approach (Karas et al. 1992) was refined in order to improve the resolution around the inner edge of the disc, very near to the $\mathrm{BH}$ horizon, and to ensure better confidence in the region where strong focusing occurs. This turned out to be necessary because the computed line profiles are very sensitive to the radius of the innermost emitting orbit, especially if the emissivity varies rapidly with radius. We recall that the innermost emission area is usually identified with a ring located at the marginally stable orbit, $r_{1} \approx r_{\mathrm{ms}}$, the value of which depending on the $\mathrm{BH}$ angular momentum.

The local emissivity law, $F(r)$, must be specified before running the code. Different choices are available: a powerlaw dependence $F_{q}(r) \propto r^{-q}$; a "standard" NovikovThorne-Page model (Novikov et al. 1974); or the effect of illumination from a central source (Martocchia \& Matt 1996). In the latter case, $h$ (the primary source height) is a phenomenological parameter which determines the form of the local emissivity law: small $h$ corresponds to enhanced concentration of primary X-rays near the horizon, resulting in substantial anisotropy of the reflected component. The local emissivity has an angular dependence, too, including possibly a limb-darkening law. This may be expressed either by the functional forms for fluorescent and Compton-reflected emission given by Ghisellini et al. (1994), or, alternatively, by the numerically-derived tables from the Montecarlo computations by Matt et al. (1991), which yielded the whole spectra of the reflected component.

The local profiles of the emissivity were interpolated by polynomials to save time machine (cp. Martocchia 2000; Martocchia et al. 2001). These emissivity laws are valid between the inner disc edge (assumed to be equal to or greater than the innermost stable orbit) and the outer edge. Because the emission tends to be concentrated in 
the central parts of the disc, the inner emitting radius is usually better constrained in the fits than the outer radius.

Typically, the code may take up to a few hours, on a common DECalpha workstation, to fit a spectrum using curved geodesics in the very vicinity of the $\mathrm{BH}$, i.e. accounting for radiation emitted at distances of the order of $r_{\mathrm{g}}$ from the event horizon.

\section{References}

Arnaud, K. A. 1996, Astronomical Data Analysis Software and Systems V, ed. G. Jacoby, \& J. Barnes, ASP Conf. Ser., 101, 17

Bardeen, J. M., Press, W. H., \& Teukolsky, S. A. 1972, ApJ, 178,347

Belloni, T., Mendez, M., King, A. R., van der Klis, M., \& van Paradijs, J. 1997a, ApJ, 479, L145

Belloni, T., Mendez, M., King, A. R., van der Klis, M., \& van Paradijs, J. 1997b, ApJ, 488, L109

Belloni, T., Klein-Wolt, M., Mendez, M., van der Klis, M., \& van Paradijs, J. 2000, A\&A, 355, 271

Boella, G., et al. 1997, A\&AS, 122, 299

Castro-Tirado, A. J., Brandt, S., \& Lund, N. 1992, IAU Circ., 5590

Cui, W., Zhang, S. N., \& Chen, W. 1998, ApJ, 492, L53

Fabian, A. C., Rees, M. J., Stella, L., \& White, N. E. 1989, MNRAS, 238, 729

Fender, R. P., et al. 1999, MNRAS, 304, 865

Feroci, M., et al. 1999, A\&A, 351, 985

Frontera, F., et al. 2001, ApJ, 546, 1027

Ghisellini, G., Haardt, F., \& Matt, G. 1994, MNRAS, 267, 743

Gierliński, M., et al. 1999, MNRAS, 309, 496

Gierliński, M., Maciołek-Niedźwiecki, A., \& Ebisawa, K. 2001, MNRAS, 325, 1253

Greiner, J., Morgan, E., \& Remillard, R. A. 1996, ApJ, 473, L107

Greiner, J., et al. 2001a, A\&A, 373, L37

Greiner, J., Cuby, J. G., \& McCaughrean, M. J. 2001b, Nature, 414,522

Karas, V., Vokrouhlický, D., \& Polnarev, A. G. 1992, MNRAS, 259,569

Laor, A. 1991, ApJ, 376, 90
Lee, J. C., et al. 2001, ApJ, in press [astro-ph/0111132]

Magdziarz, P., \& Zdziarski, A. A. 1995, MNRAS, 273, 837

Markwardt, C. B., Swank, J. H., \& Taam, R. E. 1999, ApJ, 513, L37

Martocchia, A. 2000, X-ray Spectral Signatures of Accreting Black Holes, Ph.D. Thesis, SISSA-ISAS, Trieste

Martocchia, A., \& Matt, G. 1996, MNRAS, 282, L53

Martocchia, A., Matt, G., \& Karas, V. 2001, in Proc. of the X-Ray Astronomy '999 Meeting, held in Bologna, 6-10/9/1999, Astroph. Lett. Comm., in press

Martocchia, A., Karas, V., \& Matt, G. 2000, MNRAS, 312, 817

Matt, G., Perola, G. C., \& Piro, L. 1991, A\&A, 247, 25

Matt, G., Perola, G. C., Piro, L., \& Stella, L. 1992, A\&A, 257, 63; Erratum in: A\&A, 263, 453

Matt, G., Fabian, A. C., \& Ross, R. R. 1993, MNRAS, 262, 179

Matt, G., Fabian, A. C., \& Ross, R. R. 1996, MNRAS, 278, 1111

Merloni, A., Fabian, A. C., \& Ross, R. R. 2000, MNRAS, 313, 193

Merloni, A., Vietri, M., Stella, L., \& Bini, D. 1999, MNRAS, 304,155

Miller, J. M., Fox, D. W., Di Matteo, T., et al. 2001, ApJ, 546, 1055

Miller, J. M., et al. 2002a, ApJ, submitted [astro-ph/0202083]

Miller, J.M., et al. 2002b, ApJL, submitted [astro-ph/0202375]

Mirabel, I. F., \& Rodriguez, L. F. 1994, Nature, 371, 46

Morgan, E. H., Remillard, R. A., \& Greiner, J. 1997, ApJ, 482, 993

Novikov, I. D., Thorne, K. S., \& Page, D. N. 1974, ApJ, 191

Nowak, M. A., Wagoner, R. V., Begelman, M. C., \& Lehr, D. E. 1997, ApJ, 477, L91

Orosz, J. A., \& Bailyn, C. D. 1997, ApJ, 477, 876

Rodriguez, L. F., \& Mirabel, I. F. 1999, ApJ, 511, 398

Shahbaz, T., van der Hooft, F., Casares, J., Charles, P. A., \& Van Paradijs, J. 1999, MNRAS, 306, 89

Shapiro, S. L., \& Teukolsky, S. A. 1983, Black Holes, White Dwarfs, and Neutron Stars (New York: Wiley)

Stella, L., Vietri, M., \& Morsink, S. M. 1999, ApJ, 524, 63

Strohmayer, T. E. 2001, ApJ, 554, L169

Tanaka, Y., et al. 1995, Nature, 375, 659

Zhang, S. N., Cui, W., \& Chen, W. 1997, ApJ, 482, L155 